\title{
Renormalization factor of four fermi operators with clover fermion and Iwasaki gauge action
}

\section{Yusuke Taniguchi*}

Institute of Physics, University of Tsukuba, Tsukuba, Ibaraki, 305-8571, Japan

E-mail: taniqchiehet.ph.tsukuba.ac.ip

\begin{abstract}
Renormalization factors of four-quark operators are perturbatively calculated for the improved Wilson fermion with clover term and the Iwasaki gauge action. A main application shall be the $K \rightarrow \pi \pi$ decay amplitude and the calculation is restricted to the parity odd operator, for which the operators are multiplicatively renormalizable without mixing with wrong operators that have different chiral structures.
\end{abstract}

XXIX International Symposium on Lattice Field Theory

July 10 - 162011

Squaw Valley, Lake Tahoe, California

\footnotetext{
* Speaker.
} 


\section{Introduction}

Calculation of weak matrix elements of phenomenological interest is one of major application of lattice QCD. A calculation of four quark hadron matrix elements with the Wilson fermion encounters an obstacle since unwanted mixing is introduced through quantum correction with operators that have wrong chirality.

One of the solution is to make use of the parity odd operator. By using discrete symmetries of the parity, the charge conjugation and flavor exchanging transformations it was shown [U] that the parity odd four quark operator has no extra mixing with wrong operators even without chiral symmetry. One of application of this virtue may be a calculation of the $K \rightarrow \pi \pi$ decay amplitude with the Wilson fermion.

An improvement with the clover term is indivisible for the Wilson fermion. The RG improved gauge action of Iwasaki type has a good property at lattice spacing around $a^{-1} \sim 2 \mathrm{GeV}$ imitating that in the continuum. It is plausible to use a combination of the Iwasaki gauge action and the improved Wilson fermion with clover term for our numerical simulation. Unfortunately renormalization factors of the four quark operators are not available for this combination of action. A purpose of this report is to give the renormalization factor of four quark operators perturbatively which contribute to the $K \rightarrow \pi \pi$ decay.

\section{Four quark operators}

We adopt the Iwasaki gauge action and the improved Wilson fermion action with the clover term. The Feynman rules for this action is given in Ref. []]. We shall adopt the Feynman gauge and set the Wilson parameter $r=1$ in the following.

We shall evaluate the renormalization factor of the following ten operators

$$
\begin{aligned}
& Q^{(2 n-1)}=(\bar{s} d)_{L} \sum_{q=u, d, s} \alpha_{q}^{(n)}(\bar{q} q)_{L}, Q^{(2 n)}=(\bar{s} \times d)_{L} \sum_{q=u, d, s} \alpha_{q}^{(n)}(\bar{q} \times q)_{L},(n=1,2,5), \\
& Q^{(2 n-1)}=(\bar{s} d)_{L} \sum_{q=u, d, s} \alpha_{q}^{(n)}(\bar{q} q)_{R}, Q^{(2 n)}=(\bar{s} \times d)_{L} \sum_{q=u, d, s} \alpha_{q}^{(n)}(\bar{q} \times q)_{R},(n=3,4), \\
& \alpha_{q}^{(1)}=(1,0,0), \quad \alpha_{q}^{(2)}=\alpha_{q}^{(3)}=(1,1,1), \quad \alpha_{q}^{(4)}=\alpha_{q}^{(5)}=\left(1,-\frac{1}{2},-\frac{1}{2}\right)
\end{aligned}
$$

where

$$
(\bar{s} d)_{R / L}=\bar{s} \gamma_{\mu}\left(1 \pm \gamma_{5}\right) d
$$

and $\times$ means a following contraction of the color indices

$$
Q^{(2)}=(\bar{s} \times d)_{L}(\bar{u} \times u)_{L}=\left(\bar{s}_{a} d_{b}\right)_{L}\left(\bar{u}_{b} u_{a}\right)_{L} .
$$

We are interested in the parity odd part only, which contribute to the $K \rightarrow \pi \pi$ decay amplitude

$$
\begin{aligned}
& Q_{V A+A V}^{(2 n-1)}=-Q_{V A}^{(2 n-1)}-Q_{A V}^{(2 n-1)}, \quad Q_{V A+A V}^{(2 n)}=-Q_{V A}^{(2 n)}-Q_{A V}^{(2 n)}, \quad(n=1,2,5), \\
& Q_{V A-A V}^{(2 n-1)}=Q_{V A}^{(2 n-1)}-Q_{A V}^{(2 n-1)}, \quad Q_{V A-A V}^{(2 n)}=Q_{V A}^{(2 n)}-Q_{A V}^{(2 n)}, \quad(n=3,4),
\end{aligned}
$$




$$
\begin{aligned}
& Q_{V A}^{(2 n-1)}=(\bar{s} d)_{V} \sum_{q=u, d, s} \alpha_{q}^{(n)}(\bar{q} q)_{A}, \quad Q_{A V}^{(2 n-1)}=(\bar{s} d)_{A} \sum_{q=u, d, s} \alpha_{q}^{(n)}(\bar{q} q)_{V}, \\
& Q_{V A}^{(2 n)}=(\bar{s} \times d)_{V} \sum_{q=u, d, s} \alpha_{q}^{(n)}(\bar{q} \times q)_{A}, \quad Q_{A V}^{(2 n)}=(\bar{s} \times d)_{A} \sum_{q=u, d, s} \alpha_{q}^{(n)}(\bar{q} \times q)_{V},
\end{aligned}
$$

where current-current vertex means

$$
(\bar{s} d)_{V}(\bar{q} q)_{A}=\left(\bar{s} \gamma_{\mu} d\right)\left(\bar{q} \gamma_{\mu} \gamma_{5} q\right)
$$

\section{Renormalization factor in $\overline{\mathrm{MS}}$ scheme}

We renormalized the lattice bare operators $Q_{\text {lat }}^{(k)}$ to get the renormalized operator $Q_{\overline{\mathrm{MS}}}^{(k)}$. We adopt the $\overline{\mathrm{MS}}$ scheme with DRED or NDR. We notice there are two kinds of one loop corrections to the operators. One is given by gluon exchanging diagrams given in Ref. [B]] for $\Delta S=2$ operator and the other is the penguin diagrams given in Ref. [四] for $\Delta S=1$ operators.

The renormalization of the operator is given by

$$
Q_{\mathrm{MS}}^{(i)}=Z_{i j}^{g} Q_{\mathrm{lat}}^{(j)}+Z_{i}^{\mathrm{pen}} Q_{\mathrm{lat}}^{\mathrm{pen}}+Z_{i}^{\mathrm{sub}} O_{\mathrm{lat}}^{\mathrm{sub}}
$$

where $Q_{\text {lat }}^{(j)}$ is the four quark operators on the lattice, $Q_{\text {lat }}^{\text {pen }}$ is the QCD penguin operator and $O_{\text {lat }}^{\text {sub }}$ is a lower dimensional operator to be subtracted. $Z_{i j}^{g}$ comes from gluon exchanging diagrams. $Z_{i}^{\text {pen }}$ is contribution from the penguin diagram.

\subsection{Gluon exchanging diagrams}

For gluon exchanging diagram the one loop contributions are evaluated in terms of those to the quark bilinear operators by using the Fierz rearrangement and the charge conjugation [B]]. Summing up contributions from three types of diagrams [B] the one loop correction to the four quark operators is given in a form

$$
Q_{\text {one-loop }}^{(i)}=T_{i j}^{\text {lat }} Q_{\text {tree }}^{(j)}
$$

where $Q_{\text {tree }}^{(j)}=Q_{V A \pm A V}^{(j)}$ is a tree level operator. The correction factors are given by

$$
\begin{aligned}
T_{11}^{\text {lat }} & =T_{22}^{\text {lat }}=T_{33}^{\text {lat }}=T_{44}^{\text {lat }}=T_{99}^{\text {lat }}=T_{10,10}^{\text {lat }} \\
& =\frac{g^{2}}{16 \pi^{2}}\left(-\frac{N^{2}+2}{N} \ln (\lambda a)^{2}+\frac{N^{2}-2}{2 N}\left(V_{V}+V_{A}\right)+\frac{1}{2 N}\left(V_{S}+V_{P}\right)\right) \\
T_{55}^{\text {lat }} & =T_{77}^{\text {lat }}=\frac{g^{2}}{16 \pi^{2}}\left(-\frac{N^{2}-4}{N} \ln (\lambda a)^{2}+\frac{N}{2}\left(V_{V}+V_{A}\right)-\frac{1}{2 N}\left(+V_{S}+V_{P}\right)\right) \\
T_{66}^{\text {lat }} & =T_{88}^{\text {lat }}=\frac{g^{2}}{16 \pi^{2}}\left(-4 \frac{N^{2}-1}{N} \ln (\lambda a)^{2}+\frac{N^{2}-1}{2 N}\left(V_{S}+V_{P}\right)\right) \\
T_{12}^{\text {lat }} & =T_{21}^{\text {lat }}=T_{34}^{\text {lat }}=T_{43}^{\text {lat }}=T_{9,10}^{\text {lat }}=T_{10,9}^{\text {lat }}=\frac{g^{2}}{16 \pi^{2}} \frac{1}{2}\left(6 \ln (\lambda a)^{2}+V_{V}+V_{A}-V_{S}-V_{P}\right) \\
T_{56}^{\text {lat }} & =T_{78}^{\text {lat }}=\frac{g^{2}}{16 \pi^{2}} \frac{1}{2}\left(-6 \ln (\lambda a)^{2}-V_{V}-V_{A}+V_{S}+V_{P}\right)
\end{aligned}
$$


where $\lambda$ is a gluon mass introduced for an infra red regularization and the number of color is $N=3$. $V_{\Gamma}$ is a finite part in one loop correction to the bilinear operator, which is evaluated in Ref. [2] for various gauge actions.

The renormalization factor is given by taking a ratio of quantum corrections with that in the $\overline{\mathrm{MS}}$ scheme multiplied with the quark wave function renormalization factor $Z_{2}$

$$
\begin{aligned}
& Z_{i i}^{g}(\mu a)=\frac{\left(Z_{2}^{\overline{\mathrm{MS}}}\right)^{2}\left(1+T_{i i}^{\overline{\mathrm{MS}}}\right)}{\left(Z_{2}^{\mathrm{lat}}\right)^{2}\left(1+T_{i i}^{\mathrm{lat}}\right)}, \\
& Z_{i j}^{g}(\mu a)=T_{i j}^{\overline{\mathrm{MS}}}-T_{i j} \quad(i \neq j) .
\end{aligned}
$$

The correction factor in the DRED $\overline{\mathrm{MS}}$ scheme is given by

$$
\begin{aligned}
& T_{11}^{\overline{\mathrm{MS}}}=T_{22}^{\overline{\mathrm{MS}}}=T_{33}^{\overline{\mathrm{MS}}}=T_{44}^{\overline{\mathrm{MS}}}=T_{99}^{\overline{\mathrm{MS}}}=T_{10,10}^{\overline{\mathrm{MS}}}=\left(\frac{N^{2}+2}{N}\right) V^{\overline{\mathrm{MS}}}, \\
& T_{12}^{\overline{\mathrm{MS}}}=T_{21}^{\overline{\mathrm{MS}}}=T_{34}^{\overline{\mathrm{MS}}}=T_{43}^{\overline{\mathrm{MS}}}=T_{9,10}^{\overline{\mathrm{MS}}}=T_{10,9}^{\overline{\mathrm{MS}}}=-3 V^{\overline{\mathrm{MS}}}, \\
& T_{55}^{\overline{\mathrm{MS}}}=T_{77}^{\overline{\mathrm{MS}}}=\left(\frac{N^{2}-4}{N}\right) V^{\overline{\mathrm{MS}}}, \\
& T_{56}^{\overline{\mathrm{MS}}}=T_{78}^{\overline{\mathrm{MS}}}=3 V^{\overline{\mathrm{MS}}} \\
& T_{66}^{\overline{\mathrm{MS}}}=T_{88}^{\overline{\mathrm{MS}}}=4 \frac{N^{2}-1}{N} V^{\overline{\mathrm{MS}}}, \\
& V^{\overline{\mathrm{MS}}}=\frac{g^{2}}{16 \pi^{2}}\left(\log \left(\frac{\mu^{2}}{\lambda^{2}}\right)+1\right) .
\end{aligned}
$$

The same infra red regularization with the gluon mass should be adopted. The quark wave function renormalization factor $Z_{2}$ is given in Ref. [?]].

Substituting the above results we have

$$
\begin{aligned}
Z_{11}^{g}(\mu a) & =Z_{22}^{g}(\mu a)=Z_{33}^{g}(\mu a)=Z_{44}^{g}(\mu a)=Z_{99}^{g}(\mu a)=Z_{10,10}^{g}(\mu a) \\
& =1+\frac{g^{2}}{16 \pi^{2}}\left(\frac{3}{N} \ln (\mu a)^{2}+z_{11}^{g}\right), \\
Z_{55}^{g}(\mu a) & =Z_{77}^{g}(\mu a)=1+\frac{g^{2}}{16 \pi^{2}}\left(-\frac{3}{N} \ln (\mu a)^{2}+z_{55}^{g}\right), \\
Z_{66}^{g}(\mu a) & =Z_{88}^{g}(\mu a)=1+\frac{g^{2}}{16 \pi^{2}}\left(\frac{3\left(N^{2}-1\right)}{N} \ln (\mu a)^{2}+z_{66}^{g}\right), \\
Z_{12}^{g}(\mu a) & =Z_{21}^{g}(\mu a)=Z_{34}^{g}(\mu a)=Z_{43}^{g}(\mu a)=Z_{9,10}^{g}(\mu a)=Z_{10,9}^{g}(\mu a) \\
& =\frac{g^{2}}{16 \pi^{2}}\left(-3 \ln (\mu a)^{2}+z_{12}^{g}\right), \\
Z_{56}^{g}(\mu a) & =Z_{78}^{g}(\mu a)=\frac{g^{2}}{16 \pi^{2}}\left(3 \ln (\mu a)^{2}+z_{56}^{g}\right), \\
Z_{65}^{g}(\mu a) & =Z_{87}^{g}(\mu a)=\frac{g^{2}}{16 \pi^{2}} z_{65}^{g}=0 .
\end{aligned}
$$

The numerical value of the finite part is given in table $\square$ for $N=3$ as an expansion in $c_{\mathrm{SW}}$

$$
z_{i j}^{g}=z_{i j}^{g(0)}+c_{\mathrm{SW}} z_{i j}^{g(1)}+c_{\mathrm{SW}}^{2} z_{i j}^{g(2)} .
$$


The finite part for the NDR scheme is given in table $\square$. We need to subtract the evanescent operators in the $\overline{\mathrm{MS}}$ scheme, which comes from a difference of dimensionality from four for gamma matrices in operator vertex.

Table 1: Finite part $z_{i j}^{g}$ of the renormalization factor from gluon exchanging diagrams in the DRED scheme.

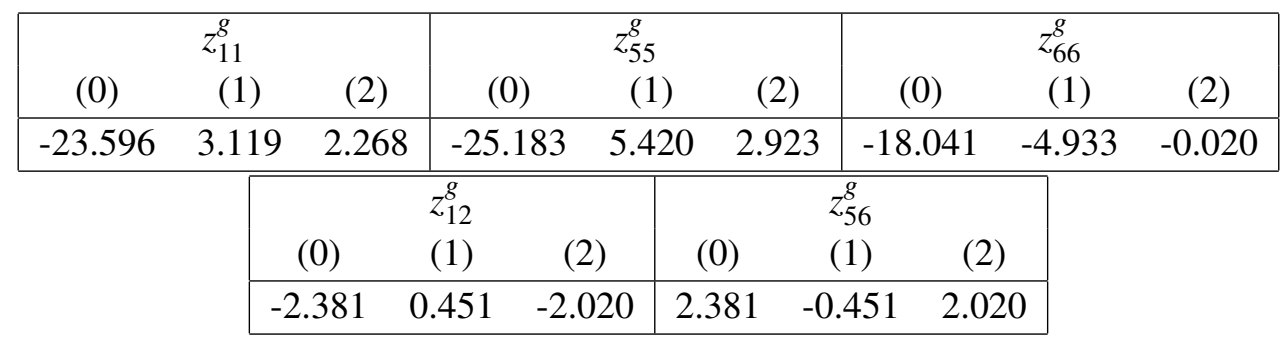

Table 2: Finite part $z_{i j}^{g(0)}$ of the renormalization factor from gluon exchanging diagrams in the NDR scheme. $c_{S W}$ dependent terms are the same as that in the DRED scheme $(n=1,2)$.

\begin{tabular}{|c|c|c|c|c|c|}
\hline$z_{11}^{g(0)}$ & $z_{55}^{g(0)}$ & $z_{66}^{g(0)}$ & $z_{12}^{g(0)}$ & $z_{56}^{g(0)}$ & $z_{65}^{g(0)}$ \\
\hline-24.096 & -25.350 & -19.708 & -4.881 & -1.120 & -3 \\
\hline
\end{tabular}

\subsection{Penguin diagrams}

Contribution from the penguin diagram is evaluated with the same procedure as in Ref. [䧃] and the one loop correction to the four quark operators is given in a form

$$
Q_{\text {one-loop }}^{(i)}=\left(T_{i}^{\text {pen }}\right)^{\text {lat }} Q_{\text {tree }}^{\text {pen }}
$$

where $Q_{\text {tree }}^{\text {pen }}$ is the penguin operator at tree level

$$
Q^{\text {pen }}=\left(Q_{V A+A V}^{(4)}+Q_{V A-A V}^{(6)}\right)-\frac{1}{N}\left(Q_{V A+A V}^{(3)}+Q_{V A-A V}^{(5)}\right) .
$$

The correction factor is given by

$$
\left(T_{i}^{\mathrm{pen}}\right)^{\text {lat }}=\frac{g^{2}}{16 \pi^{2}} \frac{C\left(Q_{i}\right)}{3}\left(\ln a^{2} p^{2}+V_{\mathrm{pen}}^{\mathrm{lat}}\right)
$$

with operator dependent factor

$$
\begin{aligned}
& C\left(Q_{1}\right)=0, \quad C\left(Q_{2}\right)=1, \quad C\left(Q_{3}\right)=2, \\
& C\left(Q_{4}\right)=C\left(Q_{6}\right)=\sum_{q=u, d, s}=N_{f}, \\
& C\left(Q_{5}\right)=C\left(Q_{7}\right)=0, \\
& C\left(Q_{8}\right)=C\left(Q_{10}\right)=\sum_{q=u, d, s} \alpha_{q}=N_{u}-\frac{N_{d}}{2}, \\
& C\left(Q_{9}\right)=-1 .
\end{aligned}
$$


$p$ is a momentum of intermediate gluon propagator given in terms of external quark momentum, for which we set the on-shell condition. The finite part is expanded as

$$
V_{\mathrm{pen}}^{\text {lat }}=-1.7128+c_{\mathrm{SW}}(-1.0878) \text {. }
$$

The correction factor in the $\overline{\mathrm{MS}}$ scheme is given in a similar form

$$
\begin{aligned}
& Q_{\text {one-loop }}^{(i)}=\left(T_{i}^{\mathrm{pen}}\right)^{\overline{\mathrm{MS}}} Q_{\mathrm{tree}}^{\mathrm{pen}}, \\
& \left(T_{i}^{\mathrm{pen}}\right)^{\overline{\mathrm{MS}}}=\frac{g^{2}}{16 \pi^{2}} \frac{C\left(Q_{i}\right)}{3}\left(\ln \left(\frac{p^{2}}{\mu^{2}}\right)-\frac{5}{3}-c\left(Q_{i}\right)\right)
\end{aligned}
$$

With the same infra red regulator $p$. The scheme dependent finite term is given by

$$
\begin{aligned}
& c^{(\mathrm{NDR})}\left(Q_{2}\right)=c^{(\mathrm{NDR})}\left(Q_{2 n-1}\right)=-1, \quad c^{(\mathrm{NDR})}\left(Q_{2 n}\right)=0, \quad(n \geq 2) \\
& c^{(\mathrm{DRED})}\left(Q_{2}\right)=c^{(\mathrm{DRED})}\left(Q_{2 n-1}\right)=c^{(\mathrm{DRED})}\left(Q_{2 n}\right)=\frac{1}{4}, \quad(n \geq 2) .
\end{aligned}
$$

Combining these two contributions the renormalization factor for the penguin operator is given by

$$
\begin{aligned}
Z_{i}^{\text {pen }} & =\left(T_{i}^{\text {pen }}\right)^{\overline{\mathrm{MS}}}-\left(T_{i}^{\mathrm{pen}}\right)^{\mathrm{lat}}=\frac{g^{2}}{16 \pi^{2}} \frac{C\left(Q_{i}\right)}{3}\left(-\ln a^{2} \mu^{2}+z_{i}^{\text {pen }}\right), \\
z_{i}^{\text {pen }} & =-V_{\text {pen }}^{\text {lat }}-\frac{5}{3}-c_{i} .
\end{aligned}
$$

Numerical value of the finite part is given in table [3].

Table 3: Finite part of the renormalization factor from the penguin diagram. Coefficients of the term $c_{S W}^{k}(k=$ $0,1)$ are given in the column marked as $(k)$.

\begin{tabular}{|l|lll|l|}
\hline$z_{i}^{\text {pen }}(\text { DRED })^{(0)}$ & $z_{2}^{\text {pen }}(\mathrm{NDR})^{(0)}$ & $z_{2 n-1}^{\text {pen }}(\mathrm{NDR})^{(0)}$ & $z_{2 n}^{\text {pen }}(\mathrm{NDR})^{(0)}$ & $\left(z_{i}^{\text {pen }}\right)^{(1)}$ \\
\hline-0.2039 & 1.0462 & 1.0462 & 0.0461 & 1.0878 \\
\hline
\end{tabular}

\subsection{Mixing with lower dimensional operator}

We shall evaluate the amputated quark bilinear vertex function given by

$$
I_{k ; X Y}^{(\mathrm{sub})}=\left\langle Q_{X Y}^{(k)} s_{a \alpha}(-p) \bar{d}_{b \beta}(p)\right\rangle_{1 \mathrm{PI}} .
$$

We consider a leading contribution to the vertex at tree level, which introduces mixing with lower dimensional operators.

We immediately get

$$
\begin{aligned}
& I_{2 n-1 ; V A}^{(\mathrm{sub})}=-I_{2 n-1 ; A V}^{(\mathrm{sub})}=\alpha_{d}^{(n)} \delta_{a b}\left(\gamma_{5}\right)_{\alpha \beta}\left(I^{(\mathrm{sub})}\left(m_{d}\right)-I^{(\mathrm{sub})}\left(m_{s}\right)\right), \\
& I_{2 n ; V A}^{(\mathrm{sub})}=-I_{2 n ; A V}^{(\mathrm{sub})}=N \alpha_{d}^{(n)} \delta_{a b}\left(\gamma_{5}\right)_{\alpha \beta}\left(I^{(\mathrm{sub})}\left(m_{d}\right)-I^{(\mathrm{sub})}\left(m_{s}\right)\right), \\
& I^{(\mathrm{sub})}(a m)=\int \frac{d^{4} l}{(2 \pi)^{4}} \frac{4 W(l, a m)}{\sin ^{2} l+W(l, a m)^{2}}, \\
& W(l, a m)=a m+\sum_{\mu}\left(1-\cos l_{\mu}\right) .
\end{aligned}
$$


which may be evaluated with an expansion in the quark mass

$$
I^{(\mathrm{sub})}(m)=\frac{1}{a^{2}} m \frac{d}{d(a m)} I^{(\mathrm{sub})}(0)+\frac{1}{a} m^{2} \frac{1}{2} \frac{d^{2}}{d(a m)^{2}} I^{(\mathrm{sub})}(0)+m^{3} \frac{1}{6} \frac{d^{3}}{d(a m)^{3}} I^{(\mathrm{sub})}(0)+\mathscr{O}(a) .
$$

The numerical value is given by

$$
\begin{aligned}
& \frac{d}{d(a m)} I^{(\mathrm{sub})}(0)=\frac{1}{16 \pi^{2}}(-21.466), \\
& \frac{d^{2}}{d(a m)^{2}} I^{(\mathrm{sub})}(0)=\frac{1}{16 \pi^{2}}(-14.92) .
\end{aligned}
$$

This contribution introduces a mixing with the lower dimensional bilinear operator $\left(\bar{s} \gamma_{5} d\right)$ multiplied with a mass difference $\left(m_{d}-m_{s}\right)$. As it is clear from (B.42) this is due to the chiral symmetry breaking effect in the Wilson fermion. It may be better not to expand in quark mass since the coefficient (3.45) is rather large and $d^{3} / d(\mathrm{am})^{3} I^{(\mathrm{sub})}(0)$ term has an infra red divergence at $m=0$. The subtraction factor is given by

$$
\begin{aligned}
& Z_{2 n-1}^{(\mathrm{sub})}=-2 \alpha_{d}^{(n)}\left(I^{(\mathrm{sub})}\left(m_{d}\right)-I^{(\mathrm{sub})}\left(m_{s}\right)\right), \quad(n=3,4), \\
& Z_{2 n}^{(\mathrm{sub})}=-2 N \alpha_{d}^{(n)}\left(I^{(\mathrm{sub})}\left(m_{d}\right)-I^{(\mathrm{sub})}\left(m_{s}\right)\right), \quad(n=3,4), \\
& Z_{2 n-1}^{(\mathrm{sub})}=Z_{2 n}^{(\mathrm{sub})}=0, \quad(n=1,2,5) .
\end{aligned}
$$

\section{Conclusion}

In this report we have calculated the one-loop contributions for the renormalization factors of parity odd four-quark operators, which contribute to the $K \rightarrow \pi \pi$ decay amplitude, in the improved Wilson fermion with clover term and the Iwasaki gauge action. The operators are multiplicatively renormalizable without any mixing with wrong operators that have different chiral structures except for the lower dimensional operator.

\section{Acknowledgment}

This work is done for a collaboration with K. -I. Ishikawa, N. Ishizuka, A. Ukawa and T. Yoshié. This work is supported in part by Grants-in-Aid of the Ministry of Education (Nos. 22540265, 23105701).

\section{References}

[1] A. Donini, V. Gimenez, G. Martinelli, M. Talevi and A. Vladikas, Eur. Phys. J. C 10 (1999) 121 [arXiv:hep-lat/9902030].

[2] S. Aoki, K. i. Nagai, Y. Taniguchi and A. Ukawa, Phys. Rev. D 58, 074505 (1998)

[arXiv:hep-lat/9802034].

[3] G. Martinelli, Phys. Lett. B141 (1984) 395.

[4] C. W. Bernard, A. Soni, T. Draper, Phys. Rev. D36 (1987) 3224. 